\title{
Editorial
}

\section{Special Series: Molecular Chaperones in Protein Folding and Disease}

ioneering work by Anfinsen established that the folding of a polypeptide is dictated by its primary sequence. ${ }^{1}$ The study of folding remains an active area of research and a question of emerging importance is how the special challenges imposed by the cytoplasm (e.g., high protein load, molecular crowding, and dynamic oligomerization) impact this process. Given the opportunities for off-pathway interactions and the threat of catastrophic aggregation, the cellular environment certainly offers unique challenges. To overcome these difficulties, cells express molecular chaperones, a family of abundant, evolutionarily conserved proteins that directly bind to protein substrates. These interactions have many consequences, including more efficient folding of nascent polypeptides and stabilization of the folding trajectory. However, chaperones also control many other aspects of a protein's life and death, including effects on aggregation, assembly into multiprotein complexes, subcellular transport, and degradation. ${ }^{2}$ In these various tasks, some chaperones appear to interact with a large percentage of the proteome, ${ }^{3}$ whereas others bind to a more restricted selection of substrates, for example, only specific components of chromatin ${ }^{4}$ or the proteasome. ${ }^{5,6}$

Of the molecular chaperones, the heat shock proteins are a powerful defense against environmental insults, such as thermal or oxidative stress. ${ }^{2}$ The heat shock proteins are typically categorized by their average molecular weight, with the major classes being Hsp40, Hsp60, Hsp70, Hsp90, Hsp104, Hsp110, and the small heat shock proteins. In the cell, members of these categories appear to play distinct roles, and consequently, they exhibit little structural or sequence homology. ${ }^{7}$ However, in response to stress, they share the feature of markedly increased expression. Moreover, many of the heat shock proteins are thought to work together toward the goal of shaping the proteome. For example, Hsp70 and Hsp90 cooperate in the folding and trafficking of nuclear hormone receptors,${ }^{8}$ and the function of many Hsp40s is thought to occur via their actions on Hsp70. In addition, it is becoming increasingly evident that the heat shock proteins are inte-

\footnotetext{
Published online in Wiley InterScience

(www.interscience.wiley.com). DOI 10.1002/bip.21290

(-2009 Wiley Periodicals, Inc.
}

grated with the ubiquitin-proteasome system, endoplasmic reticulum-associated degradation, the unfolded protein response, apoptosis signaling, the chaperone-mediated autophagy pathway and other cellular quality control systems. Collectively, these systems describe a proteostasis network, ${ }^{9}$ which regulates cellular fate through managing proteome integrity. Understanding the logic of this system will likely require more detailed knowledge of how the components are interconnected in both cells and organisms. Toward this goal, attempts to mathematically model the chaperone network are an important addition ${ }^{10,11}$ and unbiased interaction maps should also be informative. ${ }^{12}$

Beyond their roles in fundamental protein homeostasis, molecular chaperones are emerging drug targets. Many diseases are characterized by disregulation of proteostasis, including the classic examples of protein misfolding disorders, such as cystic fibrosis and Alzheimer's disease. ${ }^{13}$ Despite a clear role for aberrant protein processing in these disorders, the path to therapeutic rescue of folding balance remains uncertain. One expected benefit of a deeper understanding of chaperone biology is the ability to predict new drug targets and strategies. In support of this idea, inhibitors of the Hsp90 chaperone are currently being explored in multiple oncology clinical trials. Inhibition of Hsp90 has been shown to reduce the stability of prosurvival substrates, resulting in apoptosis of susceptible cells. ${ }^{14}$ It seems likely that other nodes within the cellular quality control network will also prove to be amenable to pharmacological targeting. However, chaperones often act on a large subset of the proteome, which means that achieving the desired therapeutic outcomes without concurrent toxicity will involve a delicate balance. In contrast, chemicals that add folding energy to specific misfolding-prone targets have also been explored in protein misfolding diseases. ${ }^{15}$ Although these strategies involve more selectivity, chemical optimization is required for each target that generates a distinct set of design challenges. It seems that a combination of strategies will likely be the best long-term scenario.

In this Special Series, experts in the field provide comprehensive updates on the most recent developments in the study of molecular chaperones, protein folding, and disease. In navigating this Special Series, one important question to consider is: what are some major, current goals of the field? 
There are certainly no "correct" answers but linking biophysical and structural studies to the cellular environment remains a challenge. Additionally, understanding the logic of the proteostasis network and the connectivity of the quality control pathways are areas of interest. How do the chaperones interact with each other and with their substrates? How can these networks be tuned to rescue diseased systems?

Paralleling the remarkable diversity of publications in this area, manuscripts within this Special Series discuss the major issues from different points of view. For example, Johnson and Zuehlke provide an excellent overview of Hsp90 biology, and how this molecular chaperone operates in the specific context of its associated cochaperones. They summarize recent evidence that combinatorial assembly of multichaperone complexes might provide more robust folding outcomes. Witt contributes an in-depth analysis of the Hsp70 chaperone, and how the structural and biochemical properties of this machine might be exploited in Parkinson's disease. $\mathrm{He}$ specifically addresses the possibilities for pharmacological intervention, and how the rationale for this approach emerged from the basic biology. Following a conceptually similar design, but on a distinct chaperone with unique biology, Lashuel and Grimminger-Marquardt provide a comprehensive look at Hsp104. These authors nicely demonstrate how biophysical and structural studies of this chaperone have contributed to our understanding of protein processing. Cyr and Douglas add a fascinating look into how networks of chaperones impinge on protein aggregation and they highlight how both pro- and anti-aggregation outcomes can be beneficial. Finally, one of the emerging areas in chaperone biology is to exploit new findings for biotechnological purposes. Accordingly, Fisher and his colleagues suggest interesting, new applications of the chaperonin, GroEL. They show how GroEL can be used to both identify chemical probes and understand protein folding/misfolding propensities. Together, these articles are intended to provide an overview of the major chaperone systems, their connections, and their potential roles in disease.

JASON E. GESTWICKI Department of Pathology and the Life Sciences Institute University of Michigan 210 Washtenaw Ave Ann Arbor, MI 48109-2216

\section{REFERENCES}

1. Anfinsen, C. B. Science 1973, 181, 223-230.

2. Bukau, B.; Weissman, J.; Horwich, A. Cell 2006, 125, 443-451.

3. Rudiger, S.; Germeroth, L.; Schneider-Mergener, J.; Bukau, B. EMBO J 1997, 16, 1501-1507.

4. Huang, S.; Zhou, H.; Katzmann, D.; Hochstrasser, M.; Atanasova, E.; Zhang, Z. Proc Natl Acad Sci USA 2005, 102, 13410-13415.

5. Kaneko, T.; Hamazaki, J.; Iemura, S.-I.; Sasaki, K.; Furuyama, K.; Natsume, T.; Tanaka, K.; Murata, S. Cell 2009, 137, 914-925.

6. Funakoshi, M.; Tomko, R. J.; Kobayashi, H.; Hochstrasser, M. Cell 2009, 137, 887-899.

7. Vos, M. J.; Hageman, J.; Carra, S.; Kampinga, H. H. Biochemistry 2008, 47, 7001-7011.

8. Pratt, W. B.; Morishima, Y.; Murphy, M.; Harrell, M. Handb Exp Pharmacol 2006, 172, 111-138.

9. Balch, W. E.; Morimoto, R. I.; Dillin, A.; Kelly, J. W. Science 2008, 319, 916-919.

10. Hu, B.; Mayer, M. P.; Tomita, M. Biophys J 2006, 91, 496-507.

11. Wiseman, R. L.; Powers, E. T.; Buxbaum, J. N.; Kelly, J. W.; Balch, W. E. Cell 2007, 131, 809-821.

12. Gong, Y.; Kakihara, Y.; Krogan, N.; Greenblatt, J. F.; Emili, A.; Zhang, Z.; Houry, W. A. Mol Syst Biol 2009, 5, 1-14.

13. Dobson, C. M. Nature 2003, 426, 884-890.

14. Neckers, L. Handb Exp Pharmacol 2006, 259-277.

15. Bernier, V.; Lagace, M.; Bichet, D. G.; Bouvier, M. Trends Endocrinol Metab 2004, 15, 222-228. 